\title{
Metabolic phenotypes in primary unknown metastatic carcinoma
}

\author{
Hye Min Kim ${ }^{1}$, Do Hee Kim ${ }^{1}$, Woo Hee Jung ${ }^{1}$ and Ja Seung Koo ${ }^{1,2^{*}}$
}

\begin{abstract}
Background: The purpose of this study is to evaluate expression of metabolism-related proteins in primary unknown metastatic carcinoma (PUMC) and associated implications for treatment.

Methods: A tissue microarray containing 77 cases of PUMC was constructed and immunohistochemical staining was used to evaluate expression of the following proteins: Glycolysis-related: Glut-1, carbonic anhydrase (CA) IX, and monocarboxylate transporter (MCT) 4; Glutaminolysis-related: glutaminase1 (GLS1), glutamate dehydrogenase (GDH), and amino acid transporter-2 (ASCT2); and Mitochondrial-related: ATP synthase, succinate dehydrogenase (SDH)A, and SDHB. The association between immunohistochemical staining results and clinicopathologic parameters was evaluated.
\end{abstract}

Results: The expression of metabolism-related proteins was different depending on the histologic subtype. Compared to other subtypes, squamous cell carcinomas (SQ) expressed more Glut-1 ( $p=0.028)$, while adenocarcinomas (AD) expressed more SDHB in the stroma $(p=0.025)$. The expression of metabolism-related proteins was also different depending on the clinical subtypes. Glut-1 was expressed most in the nodal type and the least in carcinomatosis type, when compared to other subtypes $(p=0.021)$. The metabolic phenotypes also showed other trends: when the stroma showed no glutaminolysis, the tumor mostly invaded lymph node, bone, and brain, while the tumor invaded regions other than lymph node, bone, and brain when the stroma showed glutaminolysis $(p=0.003)$. When the stroma showed the mitochondrial metabolic type, the histologic subtype was mainly $A D$, but the non-mitochondrial type was associated more with SQ $(P=0.049)$.

Conclusion: For PUMC, the expression of metabolism-related proteins, such as Glut-1 and SDHB, differs in the tumor or stroma depending on the clinical and histologic tumor subtype.

Keywords: Carcinoma, Primary unknown, Metabolism

\section{Background}

Primary unknown metastatic carcinoma (PUMC) is defined as metastatic carcinoma with no definitive primary tumor identified from clinical symptoms, patient history, radiologic imaging, laboratory investigation, histologic evaluation, or immunohistochemical staining [1]. PUMC is a heterogeneous disease which accounts for $5 \sim$ $15 \%$ of malignant tumors [2-4]; histologically, it consists of adenocarcinomas $(\mathrm{AD})(50 \sim 60 \%)$, poorly differentiated carcinomas (PD) $(30 \sim 40 \%)$, and other histologic types,

\footnotetext{
*Correspondence: kjs1976@yuhs.ac

'Department of Pathology, Yonsei University College of Medicine, Severance Hospital, 50 Yonsei-ro, Seodaemun-gu, Seoul, South Korea

2Department of Pathology, Severance Hospital, Brain Korea 21 PLUS Project

for Medical Science Yonsei University College of Medicine, Seoul

South Korea
}

including squamous cell carcinomas (SQ) $(5 \sim 8 \%)$ and undifferentiated carcinomas (UD) $(2-5 \%)[4,5]$. The exact nature of PUMC is not well defined, but one hypothesis is that it is a true metastatic tumor without an identifiable primary focus, while another hypothesis is that it is an unusual primary tumor which simulates metastatic disease [5].

In general, cancer cells show different metabolic characteristics from normal cells. Normal cells obtain energy by oxidative phosphorylation, while cancer cells use glycolysis. It is called Warburg effect theory [6]. Thus, glycolysis is one of the most important components of cancer metabolism. However, glycolysis, while a major characteristic of cancer cell metabolism, cannot account for energy usage in all types of cancer cells. According to the literature, the dominant metabolic process can be 
either glycolysis or oxidative phosphorylation based on the tumor type [7]. In addition to glucose metabolism, glutamine metabolism is also an important cancer cell metabolic pathway. Glutamine metabolism is important in that it both produces ATP and provides intermediates for macromolecular synthesis [8]. Therefore, glycolysis, glutaminolysis, and mitochondrial metabolism are likely to play indispensable roles in tumor metabolism.

There are studies about metabolism-related protein expression in various cancers. Most of the metabolismrelated protein expression differ among clinicopathologic parameter of cancer [9-14], it can be suggested that PUMC also show different expression of metabolismrelated protein expression, however there isn't enough study.

The purpose of this study is to evaluate metabolismrelated protein expression in PUMC and associated implications for treatment.

\section{Methods}

Patient selection and clinicopathologic evaluation

This study utilized formalin-fixed, paraffin-embedded (FFPE) tissue samples from patients ultimately diagnosed with PUMC at Severance Hospital. All patients were diagnosed with metastatic carcinoma by pathologists from
January 1999 to December 2012. Cases with only a small amount of biopsy material were excluded. All archival hematoxylin and eosin (H\&E)-stained slides were reviewed for each case. The clinicopathologic parameters evaluated in each tumor included patient age, sex, histological type, involved organ, and patient outcome. According to the histologic standards, PUMCs were categorized into four subtypes [5]: AD showed glandular differentiation within the tumor; SQ showed evidence of squamous differentiation, such as intercellular bridges and keratin pearls; PD did not show differentiation to any specific lineage; and UD were composed of syncytial tumor cell nests or singly scattered tumor cells closely intermingled with dense lymphoplasmacytic infiltration similar to nasopharyngeal undifferentiated carcinoma. Also, according to the clinical and radiologic diagnosis, PUMCs were categorized into subtypes: 1) "nodal type" if the tumor involved only the lymph node; 2) "single organ type" if the metastatic tumor involved only one organ (other than lymph nodes); for example, metastatic adenocarcinoma of brain or bone with unknown primary origin; 3) "intermediate type" in which two organs were involved; and 4) "carcinomatosis type" if three or more organs were involved. The study was approved by the Institutional Review Board of Severance Hospital (4-2012-0606).

Table 1 Clinicopathologic characteristics of patients

\begin{tabular}{|c|c|c|c|c|c|c|}
\hline \multirow{2}{*}{ Clinical parameters } & \multirow{2}{*}{$\begin{array}{c}\text { Total } \\
\mathrm{N}=77(\%)\end{array}$} & \multicolumn{4}{|c|}{ Histologic subtype } & \multirow[t]{2}{*}{ p-value } \\
\hline & & $A D(n=27)(\%)$ & $\mathrm{PD}(\mathrm{n}=16)(\%)$ & SQ $(n=20)(\%)$ & UD $(n=14)(\%)$ & \\
\hline Age (years, mean $\pm S D$ ) & $55.4 \pm 11.8$ & $59.3 \pm 12.3$ & $55.3 \pm 12.1$ & $55.0 \pm 9.6$ & $48.3 \pm 10.6$ & 0.042 \\
\hline Sex & & & & & & 0.521 \\
\hline Female & $29(37.7)$ & $13(48.1)$ & $6(37.5)$ & $6(30.0)$ & $4(28.6)$ & \\
\hline Male & $48(62.3)$ & $14(51.9)$ & $10(62.5)$ & $14(70.0)$ & $10(71.4)$ & \\
\hline Clinical subtype & & & & & & $<0.001$ \\
\hline Nodal type & $34(44.2)$ & $3(11.1)$ & $8(50.0)$ & $14(70.0)$ & $9(64.3)$ & \\
\hline Single organ type & $15(19.5)$ & $6(22.2)$ & $5(31.3)$ & $0(0.0)$ & $4(28.6)$ & \\
\hline Intermediate type & $14(18.2)$ & $7(25.9)$ & $1(6.3)$ & $5(25.0)$ & $1(7.1)$ & \\
\hline Carcinomatosis type & $14(18.2)$ & $11(40.7)$ & $2(12.5)$ & $1(5.0)$ & $0(0.0)$ & \\
\hline Organs involved & & & & & & 0.102 \\
\hline Lymph node & 49 (63.6) & $12(44.4)$ & $9(56.3)$ & $19(95.0)$ & $9(64.3)$ & \\
\hline Bone & $10(13.0)$ & $6(22.2)$ & $2(12.5)$ & $0(0.0)$ & $2(14.3)$ & \\
\hline Brain & $9(11.7)$ & $4(14.8)$ & $2(12.5)$ & $1(5.0)$ & $2(14.3)$ & \\
\hline Other & $9(11.7)$ & $5(18.5)$ & $3(18.8)$ & $0(0.0)$ & $1(7.1)$ & \\
\hline Postoperative treatment & & & & & & 0.039 \\
\hline None & $21(27.3)$ & $8(29.6)$ & $3(18.8)$ & $4(20.0)$ & $6(42.9)$ & \\
\hline Chemotherapy & $24(31.2)$ & $12(44.4)$ & $5(31.3)$ & $3(15.0)$ & $4(28.6)$ & \\
\hline Radiation therapy & $15(19.5)$ & $6(22.2)$ & $1(6.3)$ & $6(30.0)$ & $2(14.3)$ & \\
\hline Chemo-radiation therapy & $17(22.1)$ & $1(3.7)$ & $7(43.8)$ & $7(35.0)$ & $2(14.3)$ & \\
\hline
\end{tabular}

PD: poorly differentiated carcinoma, AD: adenocarcinoma, SQ: squamous cell carcinoma, UD: undifferentiated carcinoma. Bold represents $\mathrm{p}<0.05$. 
Tissue microarray (TMA)

Among the H\&E-stained slides, the most appropriate FFPE tumor tissue samples were gathered retrospectively, the most representative tumor area was marked, the selected area was extracted with a punch machine, and the $3 \mathrm{~mm}$ tissue core was inserted into the $6 \times 5$ recipient block. The TMA was constructed with two tissue cores for all cases.

\section{Immunohistochemistry (IHC)}

The antibodies used for IHC in this study are shown in Additional file 1: Table S1. Using xylene and alcohol solution, the $3 \mathrm{~mm}$-thick slices from the FFPE tissue block were deparaffinized and rehydrated, and then a Ventana Discovery XT automated stainer (Ventana Medical Systems, Tucson, AZ, USA) was used. Antigen retrieval was performed with $\mathrm{CC} 1$ (Cell Conditioning 1) buffer (citrate buffer $\mathrm{pH}$ 6.0, Ventana Medical Systems). IHC was performed, including the appropriate positive and negative controls. The primary antibody incubation step was omitted in the negative control. For each antibody, the positive controls recommended by the manufacturers were used.

\section{Interpretation of immunohistochemical results}

IHC result interpretation was based on the product of the proportion of stained cells and the immunhistochemical staining intensity. A product between $0-1$ was called negative and a product between 2-6 was called positive [9]. The proportion of stained cells was scored as 0 for negative, 1 for positive with less than $30 \%$, and 2 for positive with greater than or equal to than $30 \%$. Immunhistochemical staining intensity was scored as 0 for negative, 1 for weak, 2 for moderate, and 3 for strong. For the interpretation of immunohistochemical TMA, the interpretation criteria above is applied to all two cores if there were any difference in the staining.

\section{Statistical analysis}

Data were statistically processed using SPSS for Windows version 12.0 (SPSS Inc., Chicago, IL). Student's $t$ test and Fisher's exact test were used for continuous and categorical variables, respectively. Statistical significance was assumed when $\mathrm{p}<0.05$. Kaplan-Meier survival curves and log-rank statistics were employed to evaluate time to survival. Multivariate regression analysis was performed using a Cox proportional hazards model.

\section{Results}

Basal characteristics of PUMC patients according to the histologic subtypes

The basal characteristics of 77 PUMC cases, based on their histologic subtypes, are summarized in Table 1. AD accounted for 27 (35.1\%) cases, PD for 16 (20.8\%) cases, SQ for 20 (26.0\%) cases, and UD for 14 (18.2\%) cases. Depending on the histologic subtypes, the clinical subtypes were also different. While AD consisted of more of the carcinomatosis subtype, PD, SQ, and UD consisted more of the nodal subtype $(\mathrm{p}<0.001)$. Different histologic subtypes



Figure 1 Expression of metabolism-related proteins in tumor and stroma of PUMC. 
Table 2 Expression of metabolism-related proteins in PUMC according to histologic subtypes

\begin{tabular}{|c|c|c|c|c|c|c|}
\hline \multirow{2}{*}{$\begin{array}{l}\text { Immunohistochemical } \\
\text { parameters }\end{array}$} & \multirow{2}{*}{$\begin{array}{c}\text { Total } \\
\mathrm{N}=77(\%)\end{array}$} & \multicolumn{4}{|c|}{ Histologic subtype } & \multirow[t]{2}{*}{$p$-value } \\
\hline & & $A D(n=27)(\%)$ & PD $(n=16)(\%)$ & SQ $(n=20)(\%)$ & UD $(n=14)(\%)$ & \\
\hline Glut-1 (T) & & & & & & 0.028 \\
\hline Negative & $41(53.2)$ & $17(63.0)$ & $11(68.8)$ & $5(25.0)$ & $8(57.1)$ & \\
\hline Positive & $36(46.8)$ & $10(37.0)$ & $5(31.3)$ & $15(75.0)$ & $6(42.9)$ & \\
\hline Glut-1 (S) & & & & & & N/A \\
\hline Negative & $77(100.0)$ & $27(100.0)$ & $16(100.0)$ & $20(100.0)$ & $14(100.0)$ & \\
\hline Positive & $0(0.0)$ & $0(0.0)$ & $0(0.0)$ & $0(0.0)$ & $0(0.0)$ & \\
\hline CAIX (T) & & & & & & 0.124 \\
\hline Negative & $68(88.3)$ & $24(88.9)$ & $15(93.8)$ & $15(75.0)$ & $14(100.0)$ & \\
\hline Positive & $9(11.7)$ & $3(11.1)$ & $1(6.3)$ & $5(25.0)$ & $0(0.0)$ & \\
\hline CAIX (S) & & & & & & 0.050 \\
\hline Negative & 75 (97.4) & $27(100.0)$ & $14(87.5)$ & $20(100.0)$ & $14(100.0)$ & \\
\hline Positive & $2(2.6)$ & $0(0.0)$ & $2(12.5)$ & $0(0.0)$ & $0(0.0)$ & \\
\hline MCT4 (T) & & & & & & 0.409 \\
\hline Negative & $22(28.6)$ & $8(29.6)$ & $7(43.8)$ & $4(20.0)$ & $3(21.4)$ & \\
\hline Positive & $55(71.4)$ & $19(70.4)$ & $9(56.3)$ & $16(80.0)$ & $11(78.6)$ & \\
\hline MCT4 (S) & & & & & & 0.902 \\
\hline Negative & $43(55.8)$ & $15(55.6)$ & $10(62.5)$ & $10(50.0)$ & $8(57.1)$ & \\
\hline Positive & $34(44.2)$ & $12(44.4)$ & $6(37.5)$ & $10(50.0)$ & $6(42.9)$ & \\
\hline GLS1 (T) & & & & & & 0.184 \\
\hline Negative & $46(59.7)$ & $14(51.9)$ & $11(68.8)$ & $15(75.0)$ & $6(42.9)$ & \\
\hline Positive & $31(40.3)$ & $13(48.1)$ & $5(31.3)$ & $5(25.0)$ & $8(57.1)$ & \\
\hline GLS1 (S) & & & & & & 0.401 \\
\hline Negative & $68(88.3)$ & $23(85.2)$ & $13(81.3)$ & $18(90.0)$ & $14(100.0)$ & \\
\hline Positive & $9(11.7)$ & $4(14.8)$ & $3(18.8)$ & $2(10.0)$ & $0(0.0)$ & \\
\hline $\mathrm{GDH}(\mathrm{T})$ & & & & & & 0.510 \\
\hline Negative & $8(10.4)$ & $2(7.4)$ & $3(18.8)$ & $1(5.0)$ & $2(14.3)$ & \\
\hline Positive & 69 (89.6) & 25 (92.6) & $13(81.3)$ & $19(95.0)$ & $12(85.7)$ & \\
\hline GDH (S) & & & & & & 0.350 \\
\hline Negative & $58(75.3)$ & $18(66.7)$ & $11(68.8)$ & $17(85.0)$ & $12(85.7)$ & \\
\hline Positive & $19(24.7)$ & $9(33.3)$ & $5(31.3)$ & $3(15.0)$ & $2(14.3)$ & \\
\hline ASCT2 (T) & & & & & & 0.413 \\
\hline Negative & $47(61.0)$ & $15(55.6)$ & $8(50.0)$ & $15(75.0)$ & $9(64.3)$ & \\
\hline Positive & $30(39.0)$ & $12(44.4)$ & $8(50.0)$ & $5(25.0)$ & $5(35.7)$ & \\
\hline ASCT2 (S) & & & & & & 0.313 \\
\hline Negative & $73(94.8)$ & $24(88.9)$ & $16(100.0)$ & $19(95.0)$ & $14(100.0)$ & \\
\hline Positive & $4(5.2)$ & $3(11.1)$ & $0(0.0)$ & $1(5.0)$ & $0(0.0)$ & \\
\hline ATP synthase (T) & & & & & & 0.597 \\
\hline Negative & $2(2.6)$ & $1(3.7)$ & $1(6.3)$ & $0(0.0)$ & $0(0.0)$ & \\
\hline Positive & 75 (97.4) & $26(96.3)$ & 15 (93.8) & $20(100.0)$ & $14(100.0)$ & \\
\hline ATP synthase (S) & & & & & & 0.101 \\
\hline Negative & $62(80.5)$ & $18(66.7)$ & $13(81.3)$ & $19(95.0)$ & $12(85.7)$ & \\
\hline Positive & 15 (19.5) & $9(33.3)$ & $3(18.8)$ & $1(5.0)$ & $2(14.3)$ & \\
\hline
\end{tabular}


Table 2 Expression of metabolism-related proteins in PUMC according to histologic subtypes (Continued)

\begin{tabular}{|c|c|c|c|c|c|c|}
\hline $\mathrm{SDHA}(\mathrm{T})$ & & & & & & 0.208 \\
\hline Negative & $3(3.9)$ & $1(3.7)$ & $2(12.5)$ & $0(0.0)$ & $0(0.0)$ & \\
\hline Positive & $74(96.1)$ & $26(96.3)$ & 14 (87.5) & $20(100.0)$ & $14(100.0)$ & \\
\hline $\mathrm{SDHA}(\mathrm{S})$ & & & & & & 0.200 \\
\hline Negative & 59 (76.6) & 17 (63.0) & 14 (87.5) & $16(80.0)$ & $12(85.7)$ & \\
\hline Positive & 18 (23.4) & $10(37.0)$ & $2(12.5)$ & $4(20.0)$ & $2(14.3)$ & \\
\hline $\mathrm{SDHB}(\mathrm{T})$ & & & & & & 0.112 \\
\hline Negative & $28(36.4)$ & $7(25.9)$ & $9(56.3)$ & $9(45.0)$ & $3(21.4)$ & \\
\hline Positive & 49 (63.6) & $20(74.1)$ & $7(43.8)$ & $11(55.0)$ & 11 (78.6) & \\
\hline $\mathrm{SDHB}(\mathrm{S})$ & & & & & & 0.025 \\
\hline Negative & $66(85.7)$ & $19(70.4)$ & 15 (93.8) & $20(100.0)$ & $12(85.7)$ & \\
\hline Positive & $11(14.3)$ & $8(29.6)$ & $1(6.3)$ & $0(0.0)$ & $2(14.3)$ & \\
\hline
\end{tabular}

PD: poorly differentiated carcinoma, AD: adenocarcinoma, SQ: squamous cell carcinoma, UD: undifferentiated carcinoma. Bold represents $\mathrm{p}<0.05$.

indicated different postoperative treatments: $\mathrm{AD}$ cases were treated mostly with chemotherapy, PD and SQ with chemoradiation therapy, and UD with only surgery $(\mathrm{p}=0.039)$.

\section{Expression of metabolism-related proteins in PUMC} according to the histologic and clinical subtype Immunohistochemical staining on metabolism-related proteins except on Glut-1 in PUMC showed prominent expression on tumor and stroma (Figure 1), which didn't show expression on stromal compartment. Table 2 and Figure 2 show the expression of metabolism-related proteins accord- ing to histologic subtypes. Compared to other subtypes, SQ expressed more Glut-1 $(\mathrm{p}=0.028)$, while AD expressed more succinate dehydrogenase $(\mathrm{SDH}) \mathrm{B}$ in its stroma $(\mathrm{p}=$ 0.025). Also, PD expressed more carbonic anhydrase (CA) IX in stroma compared to other subtypes $(\mathrm{p}=0.050)$.

Table 3 shows the expression of metabolism-related proteins depending on clinical subtypes. The expression of Glut-1 in tumor was different for each of the clinical types: the nodal type expressed Glut-1 at the highest level, and the carcinomatosis type expressed Glut-1 at the lowest level $(\mathrm{p}=0.021)$.

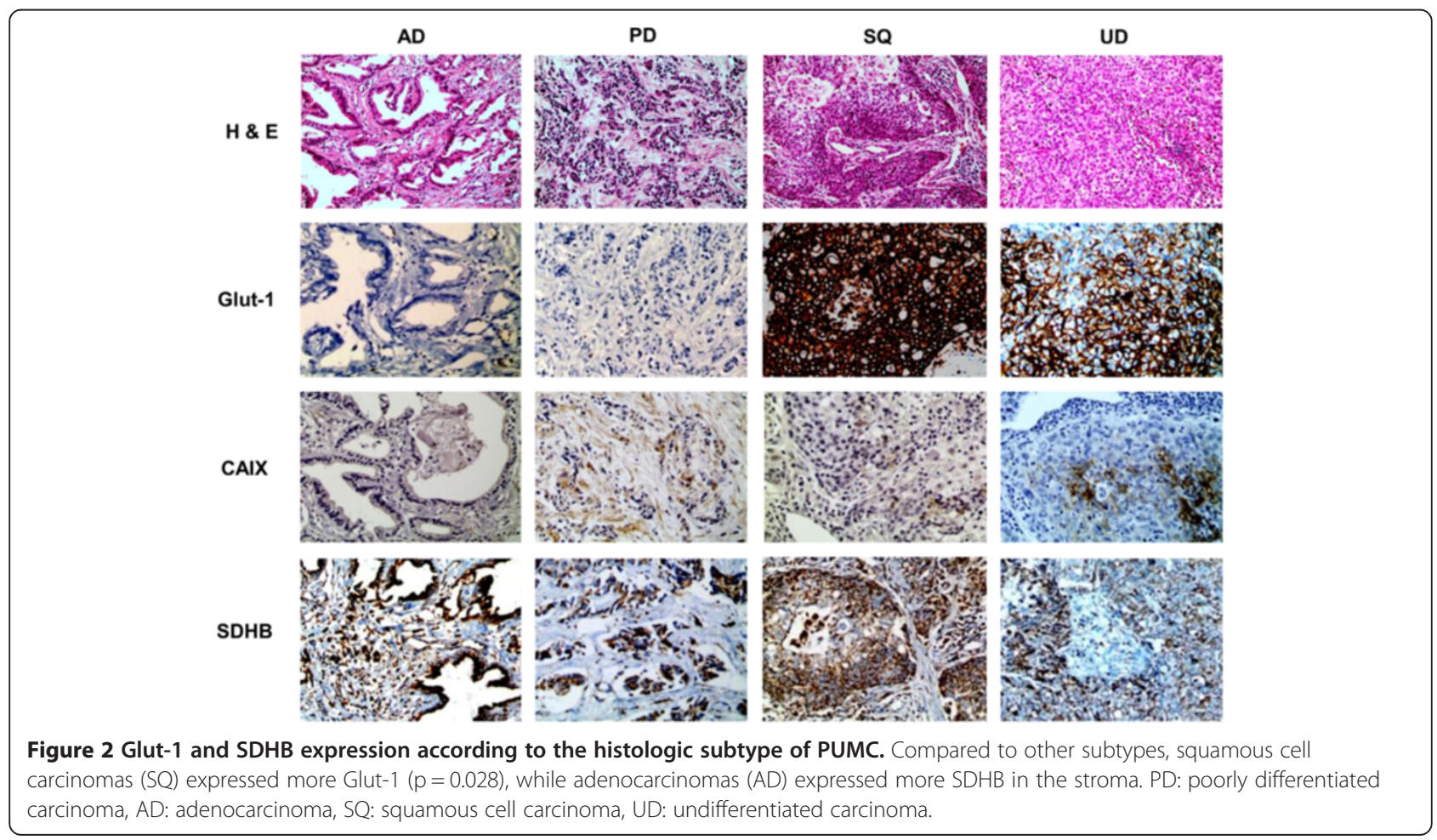


Table 3 Expression of metabolism-related proteins in PUMC according to clinical subtypes

\begin{tabular}{|c|c|c|c|c|c|c|}
\hline \multirow{2}{*}{$\begin{array}{l}\text { Immunohistochemical } \\
\text { parameters }\end{array}$} & \multirow{2}{*}{$\begin{array}{c}\text { Total } \\
(n=77)(\%)\end{array}$} & \multicolumn{4}{|c|}{ Clinical subtype } & \multirow{2}{*}{$p$-value } \\
\hline & & $\begin{array}{l}\text { Nodal type } \\
(\mathrm{n}=34)(\%)\end{array}$ & $\begin{array}{c}\text { Single organ type } \\
(n=15)(\%)\end{array}$ & $\begin{array}{c}\text { Intermediate type } \\
(n=14)(\%)\end{array}$ & $\begin{array}{l}\text { Carcinomatosis type } \\
(n=14)(\%)\end{array}$ & \\
\hline Glut-1 (T) & & & & & & 0.021 \\
\hline Negative & $41(53.2)$ & $13(38.2)$ & $9(60.0)$ & $9(64.3)$ & $10(71.4)$ & \\
\hline Positive & $36(46.8)$ & $21(61.8)$ & $6(40.0)$ & $5(35.7)$ & $4(28.6)$ & \\
\hline Glut-1 (S) & & & & & & N/A \\
\hline Negative & $77(100.0)$ & $34(100.0)$ & $15(100.0)$ & $14(100.0)$ & $14(100.0)$ & \\
\hline Positive & $0(0.0)$ & $0(0.0)$ & $0(0.0)$ & $0(0.0)$ & $0(0.0)$ & \\
\hline CAIX (T) & & & & & & 0.372 \\
\hline Negative & 68 (88.3) & $28(82.4)$ & $15(100.0)$ & $12(85.7)$ & $13(92.9)$ & \\
\hline Positive & $9(11.7)$ & $6(17.6)$ & $0(0.0)$ & $2(14.3)$ & $1(7.1)$ & \\
\hline CAIX (S) & & & & & & 0.458 \\
\hline Negative & 75 (97.4) & $33(97.1)$ & $14(93.3)$ & $14(100.0)$ & $14(100.0)$ & \\
\hline Positive & $2(2.6)$ & $1(2.9)$ & $1(6.7)$ & $0(0.0)$ & $0(0.0)$ & \\
\hline MCT4 (T) & & & & & & 0.877 \\
\hline Negative & $22(28.6)$ & $11(32.4)$ & $2(13.3)$ & $4(28.6)$ & $5(35.7)$ & \\
\hline Positive & 55 (71.4) & $23(67.6)$ & $13(86.7)$ & $10(71.4)$ & $9(64.3)$ & \\
\hline MCT4 (S) & & & & & & 0.372 \\
\hline Negative & $43(55.8)$ & $17(50.0)$ & $9(60.0)$ & $8(57.1)$ & $9(64.3)$ & \\
\hline Positive & $34(44.2)$ & $17(50.0)$ & $6(40.0)$ & $6(42.9)$ & $5(35.7)$ & \\
\hline GLS1 (T) & & & & & & 0.176 \\
\hline Negative & $46(59.7)$ & $23(67.6)$ & $8(53.3)$ & $9(64.3)$ & $6(42.9)$ & \\
\hline Positive & $31(40.3)$ & $11(32.4)$ & $7(46.7)$ & $5(35.7)$ & $8(57.1)$ & \\
\hline GLS1 (S) & & & & & & 0.123 \\
\hline Negative & $68(88.3)$ & $31(91.2)$ & $14(93.3)$ & $13(92.9)$ & $10(71.4)$ & \\
\hline Positive & $9(11.7)$ & $3(8.8)$ & $1(6.7)$ & $1(7.1)$ & $4(28.6)$ & \\
\hline GDH (T) & & & & & & 0.181 \\
\hline Negative & $8(10.4)$ & $2(5.9)$ & $2(13.3)$ & $1(7.1)$ & $3(21.4)$ & \\
\hline Positive & 69 (89.6) & $32(94.1)$ & $13(86.7)$ & $13(92.9)$ & 11 (78.6) & \\
\hline GDH (S) & & & & & & 0.816 \\
\hline Negative & $58(75.3)$ & $27(79.4)$ & $10(66.7)$ & $10(71.4)$ & $11(78.6)$ & \\
\hline Positive & $19(24.7)$ & $7(20.6)$ & $5(33.3)$ & $4(28.6)$ & $3(21.4)$ & \\
\hline $\mathrm{ASCT} 2(\mathrm{~T})$ & & & & & & 0.823 \\
\hline Negative & $47(61.0)$ & 19 (55.9) & $11(73.3)$ & $9(64.3)$ & $8(57.1)$ & \\
\hline Positive & $30(39.0)$ & $15(44.1)$ & $4(26.7)$ & $5(35.7)$ & $6(42.9)$ & \\
\hline ASCT2 (S) & & & & & & 0.255 \\
\hline Negative & $73(94.8)$ & $33(97.1)$ & $15(100.0)$ & $12(85.7)$ & $13(92.9)$ & \\
\hline Positive & $4(5.2)$ & $1(2.9)$ & $0(0.0)$ & $2(14.3)$ & $1(7.1)$ & \\
\hline ATP synthase (T) & & & & & & 0.626 \\
\hline Negative & $2(2.6)$ & $1(2.9)$ & $0(0.0)$ & $0(0.0)$ & $1(7.1)$ & \\
\hline Positive & 75 (97.4) & 33 (97.1) & $15(100.0)$ & $14(100.0)$ & $13(92.9)$ & \\
\hline ATP synthase (S) & & & & & & 0.395 \\
\hline Negative & $62(80.5)$ & $29(85.3)$ & $11(73.3)$ & $12(85.7)$ & $10(71.4)$ & \\
\hline Positive & $15(19.5)$ & $5(14.7)$ & $4(26.7)$ & $2(14.3)$ & $4(28.6)$ & \\
\hline
\end{tabular}


Table 3 Expression of metabolism-related proteins in PUMC according to clinical subtypes (Continued)

\begin{tabular}{|c|c|c|c|c|c|c|}
\hline $\mathrm{SDHA}(\mathrm{T})$ & & & & & & 0.393 \\
\hline Negative & $3(3.9)$ & $1(2.9)$ & $0(0.0)$ & $1(7.1)$ & $1(7.1)$ & \\
\hline Positive & 74 (96.1) & 33 (97.1) & 15 (100.0) & $13(92.9)$ & $13(92.9)$ & \\
\hline SDHA (S) & & & & & & 0.794 \\
\hline Negative & $59(76.6)$ & $27(79.4)$ & $11(73.3)$ & $10(71.4)$ & 11 (78.6) & \\
\hline Positive & 18 (23.4) & $7(20.6)$ & $4(26.7)$ & 4 (28.6) & $3(21.4)$ & \\
\hline $\mathrm{SDHB}(\mathrm{T})$ & & & & & & 0.230 \\
\hline Negative & $28(36.4)$ & $16(47.1)$ & $3(20.0)$ & $5(35.7)$ & $4(28.6)$ & \\
\hline Positive & 49 (63.6) & $18(52.9)$ & $12(80.0)$ & $9(64.3)$ & $10(71.4)$ & \\
\hline $\mathrm{SDHB}(\mathrm{S})$ & & & & & & 0.968 \\
\hline Negative & 66 (85.7) & $30(88.2)$ & 11 (73.3) & $13(92.9)$ & $12(85.7)$ & \\
\hline Positive & $11(14.3)$ & $4(11.8)$ & $4(26.7)$ & $1(7.1)$ & $2(14.3)$ & \\
\hline
\end{tabular}

Bold represents $p<0.05$.

Correlation between metabolic phenotypes of tumor clinicopathologic factors

When analyzing the clinicopathologic parameters based on the metabolic phenotypes based on the IHC results (Table 4), the involved organ of the PUMC was different for each mitochondrial type status. If the tumor showed a mitochondrial type, the tumor was most likely to be located at the lymph nodes $(\mathrm{p}=0.026)$.

When analyzing the clinicopathologic parameters according to the metabolic phenotypes of the stroma

Table 4 Correlation between metabolic phenotypes of tumor and clinicopathologic factors

\begin{tabular}{|c|c|c|c|c|c|c|c|c|c|}
\hline \multirow[t]{3}{*}{ Parameters } & \multicolumn{3}{|c|}{ Glycolysis type } & \multicolumn{3}{|c|}{ Glutaminolysis type } & \multicolumn{3}{|c|}{ Mitochondrial type } \\
\hline & & & p-value & & No & $p$-value & & No & $p$-value \\
\hline & $\mathrm{n}=33(\%)$ & $n=44(\%)$ & & $n=43(\%)$ & $\mathrm{n}=34(\%)$ & & $n=74(\%)$ & $\mathrm{n}=3(\%)$ & \\
\hline$\overline{\text { Age }}$ & & & 0.087 & & & 0.340 & & & 1.000 \\
\hline$<50$ & $7(21.2)$ & $18(40.9)$ & & $16(37.2)$ & $9(26.5)$ & & $24(32.4)$ & $1(33.3)$ & \\
\hline$\geq 50$ & $26(78.8)$ & $26(59.1)$ & & $27(62.8)$ & $25(73.5)$ & & $50(67.6)$ & $2(66.7)$ & \\
\hline Sex & & & 0.342 & & & 1.000 & & & 1.000 \\
\hline Male & $23(69.7)$ & $25(56.8)$ & & $27(62.8)$ & $21(61.8)$ & & $46(62.2)$ & $2(66.7)$ & \\
\hline Female & $10(30.3)$ & $19(43.2)$ & & $16(37.2)$ & $13(38.2)$ & & $28(37.8)$ & $1(33.3)$ & \\
\hline Clinical subtype & & & 0.371 & & & 0.971 & & & 0.687 \\
\hline Nodal type & $18(54.5)$ & $16(36.4)$ & & $18(41.9)$ & $16(47.1)$ & & $33(44.6)$ & $1(33.3)$ & \\
\hline Single organ type & $5(15.2)$ & $10(22.7)$ & & $9(20.9)$ & $6(17.6)$ & & $15(20.3)$ & $0(0.0)$ & \\
\hline Intermediate type & $6(18.2)$ & $8(18.2)$ & & $8(18.6)$ & $6(17.6)$ & & $13(17.6)$ & $1(33.3)$ & \\
\hline Carcinomatosis type & $4(12.1)$ & $10(22.7)$ & & $8(18.6)$ & $6(17.6)$ & & $13(17.6)$ & $1(33.3)$ & \\
\hline Histologic type & & & 0.093 & & & 0.545 & & & 0.208 \\
\hline$A D$ & $11(33.3)$ & $16(36.4)$ & & $17(39.5)$ & $10(29.4)$ & & $26(35.1)$ & $1(33.3)$ & \\
\hline PD & $4(12.1)$ & $12(27.3)$ & & $8(18.6)$ & $8(23.5)$ & & $14(18.9)$ & $2(66.7)$ & \\
\hline SQ & $13(39.4)$ & $7(15.9)$ & & $9(20.9)$ & $11(32.4)$ & & $20(27.0)$ & $0(0.0)$ & \\
\hline UP & $5(15.2)$ & $9(20.5)$ & & $9(20.9)$ & $5(14.7)$ & & $14(18.9)$ & $0(0.0)$ & \\
\hline Organs involved & & & 0.392 & & & 0.730 & & & 0.026 \\
\hline Lymph node & $24(72.7)$ & $25(56.8)$ & & $27(62.8)$ & $22(64.7)$ & & $48(64.9)$ & $1(33.3)$ & \\
\hline Bone & $3(9.1)$ & $7(15.9)$ & & $7(16.3)$ & $3(8.8)$ & & $10(13.5)$ & $0(0.0)$ & \\
\hline Brain & $4(12.1)$ & $5(11.4)$ & & $4(9.3)$ & $5(14.7)$ & & $9(12.2)$ & $0(0.0)$ & \\
\hline Other & $2(6.1)$ & $7(15.9)$ & & $5(11.6)$ & $4(11.8)$ & & $7(9.5)$ & $2(66.7)$ & \\
\hline
\end{tabular}

Bold represents $p<0.05$. 
(Table 5), the involved organ depended on the stromal glutaminolysis status; when the stroma showed no glutaminolysis, the tumor most likely had invaded lymph node, bone, and brain, while the tumor was most likely to have invaded areas other than lymph node, bone, and brain when the stroma showed glutaminolysis $(\mathrm{p}=0.003)$.

Furthermore, the histologic subtypes were also different based on the stroma mitochondrial status. When the stroma was the mitochondrial type, the histologic subtype was mainly $\mathrm{AD}$, but the non-mitochondrial type was associated more with SQ $(p=0.049)$. The cases with glycolysis-type stroma were all PD, while the cases with non-glycolysis stroma consisted of AD more often than others $(\mathrm{p}=0.050)$.

The significant correlations between metabolic phenotype status were those between glutaminolysis type $(\mathrm{T})$ and mitochondrial type $(\mathrm{T})(\mathrm{r}=0.226, \mathrm{p}=0.048)$; and glutaminolysis type $(\mathrm{S})$ and mitochondrial type $(\mathrm{S})(\mathrm{r}=0.558$, $\mathrm{p}=<0.001$, Table 6).

In analysis of correlation between metabolism-related protein expression in tumor and stroma, there was a significant correlation in SDHB (T) and SDHB (S) $(r=0.309$, $\mathrm{p}=0.006)$, SDHB (T) and SDHA (S) $(\mathrm{r}=0.290, \mathrm{p}=0.011)$, and SDHB (T) and ATP synthase $(\mathrm{S})(\mathrm{r}=0.235, \mathrm{p}=0.039)$.

\section{Correlation between the expression of glycolysis related} proteins and that of mitochondrial metabolism related proteins according to the histologic and clinical subtypes On analysis of glycolysis related protein and mitochondrial metabolism related protein expression among histologic subtype, there was a significant correlation on $\mathrm{AD}$ in SDHB (S) and MCT4 (S) $(\mathrm{r}=0.562, \mathrm{p}=0.002)$, SDHA (S) and MCT4 (S) $(\mathrm{r}=0.549, \mathrm{p}=0.003)$, and MCT4 (S) and ATP synthase (S) $(\mathrm{r}=0.632, \mathrm{p}<0.001)$, PD in SDHB (S) and CAIX (S) $(r=0.684, p=0.004)$, SQ in SDHA (S) and MCT4 (S) $(\mathrm{r}=0.500, \mathrm{p}=0.025)$, and UD in MCT4 (T) and SDHB (T) $(\mathrm{r}=0.576, \mathrm{p}=0.031)$.

On analysis of expression of glycolysis related protein and mitochondrial metabolism in clinical subtype, there was a significant correlation in nodal type in SDHB (S) and CAIX (S) $(\mathrm{r}=0.477, \mathrm{p}=0.004)$, SDHB (S) and MCT4 (S) ( $\mathrm{r}=0.365, \mathrm{p}=0.034)$, SDHA (S) and CAIX (S) $(\mathrm{r}=0.342, \mathrm{p}=0.048)$, and SDHA (S) and MCT4 (S) $(\mathrm{r}=$ $0.364, \mathrm{p}=0.034)$, in single organ type in SDHB $(\mathrm{S})$ and

Table 5 Correlation between metabolic phenotypes of stroma and clinicopathologic factors

\begin{tabular}{|c|c|c|c|c|c|c|c|c|c|}
\hline \multirow[t]{3}{*}{ Parameters } & \multicolumn{3}{|c|}{ Glycolysis type } & \multicolumn{3}{|c|}{ Glutaminolysis type } & \multicolumn{3}{|c|}{ Mitochondrial type } \\
\hline & Yes & No & $p$-value & Yes & No & p-value & Yes & No & p-value \\
\hline & $\mathrm{n}=2(\%)$ & $\mathrm{n}=75(\%)$ & & $\mathrm{n}=8(\%)$ & $\mathrm{n}=69(\%)$ & & $n=74(\%)$ & $\mathrm{n}=3(\%)$ & \\
\hline Age & & & 0.547 & & & 0.710 & & & 0.741 \\
\hline$<50$ & $1(50.0)$ & $24(32.0)$ & & $3(37.5)$ & $22(31.9)$ & & $3(25.0)$ & $22(33.8)$ & \\
\hline$\geq 50$ & $1(50.0)$ & $51(68.0)$ & & $5(62.5)$ & $47(68.1)$ & & $9(75.0)$ & $43(66.2)$ & \\
\hline Sex & & & 0.524 & & & 0.466 & & & 0.351 \\
\hline Male & $2(100.0)$ & $46(61.3)$ & & $4(50.0)$ & $44(63.8)$ & & $6(50.0)$ & $42(64.6)$ & \\
\hline Female & $0(0.0)$ & $29(38.7)$ & & $4(50.0)$ & $25(36.2)$ & & $6(50.0)$ & $23(35.4)$ & \\
\hline Clinical subtype & & & 0.627 & & & 0.857 & & & 0.533 \\
\hline Nodal type & $1(50.0)$ & $33(44.0)$ & & $3(37.5)$ & $31(44.9)$ & & $5(41.7)$ & $29(44.6)$ & \\
\hline Single organ type & $1(50.0)$ & $14(18.7)$ & & $1(12.5)$ & $14(20.3)$ & & $4(33.3)$ & $11(16.7)$ & \\
\hline Intermediate type & $0(0.0)$ & $14(18.7)$ & & $2(25.0)$ & $12(17.4)$ & & $1(8.3)$ & $13(20.0)$ & \\
\hline Carcinomatosis type & $0(0.0)$ & $14(18.7)$ & & $2(25.0)$ & $12(17.4)$ & & $2(16.7)$ & $12(18.5)$ & \\
\hline Histologic type & & & 0.050 & & & 0.237 & & & 0.049 \\
\hline$A D$ & $0(0.0)$ & $27(36.0)$ & & $5(62.5)$ & $22(31.9)$ & & $8(66.7)$ & $19(29.2)$ & \\
\hline PD & $2(100.0)$ & $14(18.7)$ & & $2(25.0)$ & $14(20.3)$ & & $2(16.7)$ & $14(21.5)$ & \\
\hline SQ & $0(0.0)$ & $20(26.7)$ & & $1(12.5)$ & 19 (27.5) & & $0(0.0)$ & $20(30.8)$ & \\
\hline UP & $0(0.0)$ & 14 (18.7) & & $0(0.0)$ & $14(20.3)$ & & $2(16.7)$ & $12(18.5)$ & \\
\hline Organs involved & & & 0.439 & & & 0.003 & & & 0.121 \\
\hline Lymph node & $1(50.0)$ & $48(64.0)$ & & $4(50.0)$ & $45(65.2)$ & & $6(50.0)$ & $43(66.2)$ & \\
\hline Bone & $1(50.0)$ & $9(12.0)$ & & $0(0.0)$ & $10(14.5)$ & & $3(25.0)$ & $7(10.8)$ & \\
\hline Brain & $0(0.0)$ & $9(12.0)$ & & $0(0.0)$ & $9(13.0)$ & & $0(0.0)$ & $9(13.8)$ & \\
\hline Other & $0(0.0)$ & $9(12.0)$ & & $4(50.0)$ & $5(7.2)$ & & $3(25.0)$ & $6(9.2)$ & \\
\hline
\end{tabular}

Bold represents $p<0.05$. 
Table 6 Correlation among metabolic phenotypes in tumor and stroma

\begin{tabular}{|c|c|c|c|c|c|c|}
\hline & $\begin{array}{l}\text { Glycolysis } \\
\text { type }(T)\end{array}$ & $\begin{array}{l}\text { Glutaminolysis } \\
\text { type }(\mathrm{T})\end{array}$ & $\begin{array}{l}\text { Mitochondrial } \\
\text { type (T) }\end{array}$ & $\begin{array}{l}\text { Glycolysis } \\
\text { type (S) }\end{array}$ & $\begin{array}{l}\text { Glutaminolysis } \\
\text { type (S) }\end{array}$ & $\begin{array}{l}\text { Mitochondrial } \\
\text { type (S) }\end{array}$ \\
\hline \multicolumn{7}{|l|}{ Glycolysis type (T) } \\
\hline Correlation coefficient & & -0.075 & 0.175 & -0.141 & -0.037 & -0.083 \\
\hline p-value & & 0.514 & 0.129 & 0.220 & 0.750 & 0.475 \\
\hline \multicolumn{7}{|l|}{ Glutaminolysis type (T) } \\
\hline Correlation coefficient & & & 0.226 & 0.145 & 0.046 & 0.094 \\
\hline$p$-value & & & 0.048 & 0.208 & 0.693 & 0.418 \\
\hline \multicolumn{7}{|l|}{ Mitochondrial type (T) } \\
\hline Correlation coefficient & & & & 0.033 & -0.151 & 0.087 \\
\hline$p$-value & & & & 0.777 & 0.189 & 0.454 \\
\hline \multicolumn{7}{|l|}{ Glycolysis type (S) } \\
\hline Correlation coefficient & & & & & 0.212 & 0.155 \\
\hline$p$-value & & & & & 0.064 & 0.178 \\
\hline \multicolumn{7}{|l|}{ Glutaminolysis type (S) } \\
\hline Correlation coefficient & & & & & & 0.558 \\
\hline $\mathrm{p}$-value & & & & & & $<0.001$ \\
\hline
\end{tabular}

T, tumor, S, stroma.

Bold represents $\mathrm{p}<0.05$.

MCT4 (S) ( $\mathrm{r}=0.739, \mathrm{p}=0.002)$, SDHA (S) and MCT4 (S) $(\mathrm{r}=0.739, \mathrm{p}=0.002)$, CAIX $(\mathrm{S})$ and SDHB $(\mathrm{T})(\mathrm{r}=-0.535$, $\mathrm{p}=0.040)$, and MCT4 $(\mathrm{S})$ and ATP synthase $(\mathrm{S})(\mathrm{r}=0.739$, $\mathrm{p}=0.002)$, in carcinomatosis type in SDHB (S) and MCT4 (S) $(\mathrm{r}=0.548, \mathrm{p}=0.043), \operatorname{SDHB}(\mathrm{S})$ and MCT4 $(\mathrm{T})$ $(\mathrm{r}=-0.548, \mathrm{p}=0.043)$, and MCT4 $(\mathrm{S})$ and ATP synthase $(\mathrm{S})(\mathrm{r}=0.849, \mathrm{p}<0.001)$, which was absent in intermediate type.

\section{Impact of clinicopathologic and immunohistochemical factors on prognosis}

Analyzing the influence of clinicopathologic factors and the expression of metabolism-related proteins on the prognosis with univariate analysis, the factors associated with shorter overall survival were histologic subtype (UD > SQ > PD > AD, $\mathrm{p}=0.002$ ) and clinical type (single organ type $>$ nodal type $>$ intermediate type $>$ carcinomatosis type, $\mathrm{p}<0.001$, Table 7 ), but the expression of metabolism-related proteins was not significantly related to the prognosis (Tables 8,9 ).

The influence of metabolism-related protein expression on the prognosis in each subgroup, categorized based on histologic subtype, was analyzed with univariate analysis. For SQ, tumoral glutamate dehydrogenase $(\mathrm{GDH})$ negativity $(\mathrm{p}=0.011)$ and stromal ATP synthase positivity $(\mathrm{p}<0.001)$ were associated with shorter overall survival (Figure 3).

\section{Discussion}

This study investigated for the metabolic features of PUMC in 77 cases. The expression of metabolism-related proteins was different for each of the histologic subtypes: tumoral Glut-1 expression was highest in SQ $(\mathrm{p}=0.028)$. Because the metabolic features of PUMC have not been commonly reported in the literature, it is difficult to draw definitive conclusions. However, Glut-1, which is thought to function as a glucose transporter during glycolysis, is expressed the more vigorously in tumors with more aggressive tumor characteristics, such as high grade, high proliferative activity, and poor prognosis [10-17]. It can be deduced that SQ is likely the tumor with greatest glycolytic activity as it expresses Glut-1 the most among all the subtypes of PUMC. The expression of Glut-1 in SQ invading organs in cases other than PUMC is reported in cases of head and neck cancer $[18,19]$ and esophageal cancer [13]. The expression of Glut-1 is associated with deep invasion, lymph node metastasis, and poor prognosis $[13,18,19]$. Glut-1 expression is also correlated to tumor aggressiveness in SQ.

In this study, stromal SDHB was expressed more in AD $(p=0.025)$. It is interesting that the degree of metabolismrelated protein expression in the stroma near the tumor differed according to the histologic tumor subtypes. Some studies have clarified that there is an interaction between tumor and stroma with respect to tumor metabolism. The representative theory is the reverse-Warburg effect theory, which says that lactate created by glycolysis in the stroma is transferred to tumor cells and converted into energy through the TCA cycle by the mitochondria [20]. Consequently, PUMC can manifest an interaction between tumor and stroma. In this study, AD showed higher expression of mitochondrial markers, such as SDHD, in 
Table 7 The impact of clinicopathologic parameters on prognosis by univariate analysis

\begin{tabular}{lllll}
\hline \multirow{2}{*}{$\begin{array}{l}\text { Clinicopathologic } \\
\text { parameters }\end{array}$} & \multicolumn{2}{c}{$\begin{array}{c}\text { No. of patients } \\
\left(\mathrm{n}=59^{*}\right)(\%)\end{array}$} & & Overall survival \\
\cline { 2 - 3 } \cline { 5 - 6 } & $\begin{array}{c}\text { No. of } \\
\text { cases }\end{array}$ & $\begin{array}{c}\text { Patient } \\
\text { death }\end{array}$ & $\begin{array}{c}\text { Median survival } \\
(95 \% \text { Cl) (months) }\end{array}$
\end{tabular}

\begin{tabular}{lccc}
\hline Age & & & \\
$<50$ & 20 & 5 & $96(70-123)$ \\
$\geq 50$ & 39 & 13 & $43(30-56)$ \\
Sex & & & \\
Male & 35 & 10 & $88(65-110)$ \\
Female & 24 & 8 & $74(44-103)$ \\
Histologic subtype & & & \\
AD & 21 & 11 & $23(10-36)$ \\
PD & 13 & 1 & $34(28-40)$ \\
SQ & 17 & 5 & $49(33-65)$ \\
UD & 8 & 1 & $115(86-145)$
\end{tabular}

Clinical subtype

$\begin{array}{lccc}\begin{array}{l}\text { Nodal type } \\ \text { Single organ } \\ \text { type }\end{array} & 25 & 3 & 107(88-126) \\ \begin{array}{l}\text { Intermediate } \\ \text { type }\end{array} & 10 & 4 & 21(9-32) \\ \quad \begin{array}{l}\text { Carcinomatosis } \\ \text { type }\end{array} & 14 & 10 & 6(3-10) \\ \text { Organs involved } & & & \\ \text { Lymph node } & 38 & 12 & 77(55-99) \\ \text { Bone } & 6 & 2 & 40(18-62) \\ \text { Brain } & 7 & 1 & 31(23-39) \\ \text { Other } & 8 & 3 & 83(40-126)\end{array}$

Postoperative

treatment

\begin{tabular}{llll} 
None & 7 & 1 & $107(75-139)$ \\
$\quad$ Chemotherapy & 20 & 7 & $77(45-108)$ \\
$\quad \begin{array}{l}\text { Radiation } \\
\text { therapy }\end{array}$ & 15 & 4 & $52(36-68)$ \\
$\quad \begin{array}{l}\text { Chemo-radiation } \\
\text { therapy }\end{array}$ & 17 & 6 & $25(18-33)$ \\
\hline
\end{tabular}

PD: poorly differentiated carcinoma, AD: adenocarcinoma, SQ: squamous cell carcinoma, UD: undifferentiated carcinoma.

"Out of 77 patients, clinical follow-up data were available in 59 patients.

Bold represents $p<0.05$.

stroma, and the ratio of mitochondrial-type stroma, defined based on the expression of mitochondrial related proteins, was higher in AD than in SQ. Further study of the metabolic interaction between tumor and stroma in PUMC with different histologic subtypes will be critical.

In this study, the expression of Glut-1 in tumor varied for each of the clinical subtypes; the nodal type showed the highest expression, but the carcinomatosis type showed the least $(\mathrm{p}=0.021)$. According to the literature,
Table 8 The impact of expression status of metabolismrelated proteins on prognosis by univariate analysis

\begin{tabular}{lcclll}
\hline $\begin{array}{l}\text { Clinicopathologic } \\
\text { parameters }\end{array}$ & \multicolumn{2}{c}{$\begin{array}{c}\text { No. of patients } \\
(\mathbf{n}=\mathbf{5 9 *})(\%)\end{array}$} & & \multicolumn{2}{c}{ Overall survival } \\
\cline { 2 - 3 } \cline { 5 - 6 } & $\begin{array}{c}\text { No. of } \\
\text { cases }\end{array}$ & $\begin{array}{c}\text { Patient } \\
\text { death }\end{array}$ & & $\begin{array}{c}\text { Median survival } \\
(\mathbf{9 5 \%} \text { Cl) (months) }\end{array}$ & P -value \\
\hline Glut-1 (T) & & & & & 0.101
\end{tabular}

Negative

$34 \quad 13$

Positive

5

Glut-1 (S)

N/

Negative

$59 \quad 18$

A

Positive

0

CAIX (T)

Negative

$51 \quad 17$

Positive

81

CAIX (S)

Negative

57

Positive

2

MCT4 (T)

Negative

Positive

40

MCT4 (S)

Negative

Positive

$\begin{array}{ll}34 & 9 \\ 25 & 9\end{array}$

GLS1 (T)

Negative

Positive

39

10

GLS1 (S)

Negative

Positive

GDH (T)

Negative

Positive

$$
20
$$

8

$\mathrm{GDH}(\mathrm{S})$

Negative

Positive

AscT2 (T)

Negative

Positive

ASCT2 (S)

$$
\text { Negative }
$$

Positive

ATP synthase (T)

Negative

Positive

2
57

57

$57 \quad 17$

ATP synthase (S)
74 (49-98)

55 (42-68)

N/A

84 (66-102)

N/A

0.272

82 (63-101)

54 (30-79)

N/A

N/A

N/A

87 (59-115)

80 (57-103)

94 (73-115)

43 (29-57)

0.139

49 (38-60)

73 (42-103)

0.721

84 (65-104)

46 (22-69) /A

72

2

/A

28 (14-39)

85 (66-104)

90 (72-109)

40 (19-60)

78 (54-101)

$36(27-45)$

87 (68-105)

$16(1-32)$

$10(10-10)$

85 (67-104) 
Table 8 The impact of expression status of metabolismrelated proteins on prognosis by univariate analysis (Continued)

\begin{tabular}{|c|c|c|c|c|}
\hline Negative & 48 & 14 & $88(68-107)$ & \\
\hline Positive & 11 & 4 & $23(12-34)$ & \\
\hline SDHA (T) & & & & 0.906 \\
\hline Negative & 3 & 1 & $28(13-43)$ & \\
\hline Positive & 56 & 17 & $84(66-103)$ & \\
\hline $\mathrm{SDHA}(\mathrm{S})$ & & & & 0.588 \\
\hline Negative & 44 & 13 & $90(70-109)$ & \\
\hline Positive & 15 & 5 & $37(15-58)$ & \\
\hline $\mathrm{SDHB}(\mathrm{T})$ & & & & 0.166 \\
\hline Negative & 23 & 5 & $86(56-115)$ & \\
\hline Positive & 36 & 13 & $79(57-102)$ & \\
\hline SDHB (S) & & & & 0.923 \\
\hline Negative & 51 & 16 & $84(65-103)$ & \\
\hline Positive & 8 & 2 & $27(16-37)$ & \\
\hline
\end{tabular}

"Out of 77 patients, clinical follow-up data were available in 59 patients.

Glut-1 is strongly related to lymph node metastasis of tumors $[21,22]$, so the higher expression in nodal type cases coincides with this data. Unusually, for the cases with extensive tumor involvement, including carcinomatosis type, the expression of Glut-1 was relatively low. One probable explanation is that the carcinomatosis type of PUMC may have discrete metabolic characteristics when compared to the carcinomatosis type of tumor with known primary cancer. When the primary carcinoma is known, the ratio of distant metastasis to more than 3 organs is less than 15\%, but this ratio for PUMC is about 30\% [23]. Moreover, the invaded organs in the PUMC cases are unusual locations, including kidney, adrenal gland, skin, and heart [24-26]. Thus, the carcinomatosis type of PUMC and primary carcinoma could be two very distinct disease entities with very different metabolic natures. Further study is required to substantiate this possibility. Another probable explanation is that the change in the metabolic characteristics for endstage cancer. In previous studies, FDG-PET CT uptake clearly correlated to the expression of Glut-1 in tissue in early-stage cancer, while the correlation was poor for late-stage cancer [27]. While requiring further study, it can be speculated that metabolic characteristics change as a tumor progresses on to later stages.

In this study, tumoral GDH negativity and stromal ATP synthase positivity showed correlation to shorter OD. It is difficult to compare, since there is no previous study on PUMC and its metabolic characteristic. However, there are several studies reporting metabolismrelated protein expression showing poor prognosis in various cancers [9-14]. Stromal GDH negativity was correlated to poor prognosis, especially in breast cancer
Table 9 The impact of metabolic phenotypes on prognosis by univariate analysis

\begin{tabular}{|c|c|c|c|c|}
\hline \multirow{2}{*}{$\begin{array}{l}\text { Clinicopathologic } \\
\text { parameters }\end{array}$} & \multicolumn{2}{|c|}{$\begin{array}{l}\text { No. of patients } \\
\left(n=59^{*}\right)(\%)\end{array}$} & \multicolumn{2}{|c|}{ Overall survival } \\
\hline & $\begin{array}{l}\text { No. of } \\
\text { cases }\end{array}$ & $\begin{array}{l}\text { Patient } \\
\text { death }\end{array}$ & $\begin{array}{c}\text { Median survival } \\
(95 \% \mathrm{Cl}) \text { (months) }\end{array}$ & $P$-value \\
\hline $\begin{array}{l}\text { Tumor metabolic } \\
\text { type }\end{array}$ & & & & 0.279 \\
\hline Glycolysis type & 22 & 5 & $52(37-67)$ & \\
\hline $\begin{array}{l}\text { Non-glycolysis } \\
\text { type }\end{array}$ & 37 & 13 & $79(56-102)$ & \\
\hline $\begin{array}{l}\text { Tumor metabolic } \\
\text { type }\end{array}$ & & & & 0.644 \\
\hline $\begin{array}{l}\text { Glutaminolysis } \\
\text { type }\end{array}$ & 30 & 10 & $84(60-108)$ & \\
\hline $\begin{array}{l}\text { Non- } \\
\text { glutaminolysis type }\end{array}$ & 29 & 8 & $45(30-59)$ & \\
\hline $\begin{array}{l}\text { Tumor metabolic } \\
\text { type }\end{array}$ & & & & 0.906 \\
\hline $\begin{array}{l}\text { Mitochondrial } \\
\text { type }\end{array}$ & 56 & 17 & $84(66-103)$ & \\
\hline $\begin{array}{l}\text { Non- } \\
\text { mitochondrial type }\end{array}$ & 3 & 1 & $28(13-43)$ & \\
\hline $\begin{array}{l}\text { Stroma metabolic } \\
\text { type }\end{array}$ & & & & $\mathrm{n} / \mathrm{a}$ \\
\hline Glycolysis type & 2 & 0 & $\mathrm{n} / \mathrm{a}$ & \\
\hline $\begin{array}{l}\text { Non-glycolysis } \\
\text { type }\end{array}$ & 57 & 18 & $\mathrm{n} / \mathrm{a}$ & \\
\hline $\begin{array}{l}\text { Stroma metabolic } \\
\text { type }\end{array}$ & & & & 0.363 \\
\hline $\begin{array}{l}\text { Glutaminolysis } \\
\text { type }\end{array}$ & 7 & 1 & $62(43-80)$ & \\
\hline $\begin{array}{l}\text { Non- } \\
\text { glutaminolysis type }\end{array}$ & 52 & 17 & $80(60-100)$ & \\
\hline $\begin{array}{l}\text { Stroma metabolic } \\
\text { type }\end{array}$ & & & & 0.864 \\
\hline $\begin{array}{l}\text { Mitochondrial } \\
\text { type }\end{array}$ & 9 & 2 & $28(18-37)$ & \\
\hline $\begin{array}{l}\text { Non- } \\
\text { mitochondrial type }\end{array}$ & 50 & 16 & $83(64-103)$ & \\
\hline
\end{tabular}

" Out of 77 patients, clinical follow-up data were available in 59 patients.

[28], which showed compatible result with this study. In addition, ATP synthase expression in stroma showed relationship with poor prognosis, and the previous studies reported metabolism related protein and poor prognosis. Especially in breast cancer, the expression of glycolysisrelated protein in stroma showed poor clinical outcome [20]. These stromal cells showing metabolic activities are defined as cancer-associated fibroblast, which show deprivement of cavelin-1 [20,29]. In PUMC, in contrast to breast cancer, ATP synthase expression, which is related to oxidative phosphorylation expression showed correlation to prognosis, which requires further study for nature of ATP synthase positive stromal cell. 

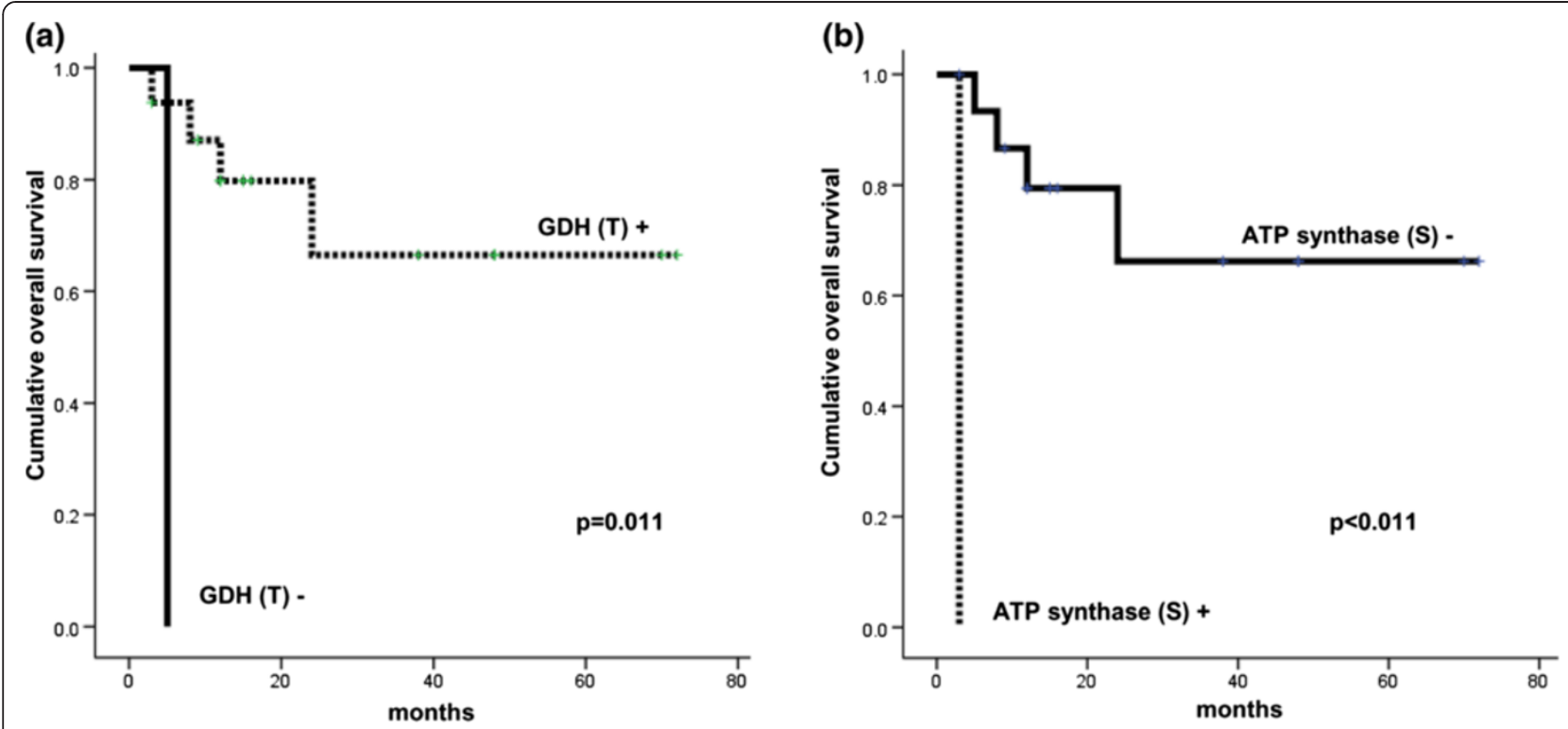

Figure 3 Overall survival curves according to tumoral GDH (a) and stromal ATP synthase (b) status in the SQ group of PUMC. T, tumor, S, stroma.

The clinical significance of this study should be focused on two. First, its significance as a predictive factor. Even though small amount of biopsy tissue shows Glut1 and/or SDHB expression in PUMC and predicts clinical, histologic subtype, prognosis by GDH, and/or ATP synthase expression in SQ type of PUMC, it needs further validation study. Secondly, its possibility as a treatment target in PUMC. Metabolism inhibitors, such as HIF-1 $\alpha$ inhibitor [30,31], Glut-1 inhibitor [32,33], CAIX inhibitor [34], and monocarboxylate transporter (MCT)4 inhibitor [35], are reported in preclinical studies to suppress tumor growth in several tumor types. In this study, only the expression of metabolism-related protein on PUMC tissue is performed. Further validation study, including in vitro cell line study, mouse xenograft study, and clinical trial, is required to verify the possibility of metabolism inhibitor, especially Glut-1 inhibitor in target therapy of PUMC.

The main limitation of this study can be the small number of cases, and too various subtypes resulting in a small number for each classifications. Thus, there needs to be further study with case expansion. However, considering previous studies on PUMC studies with the case number of $26 \sim 100$, the case number of this study does not necessarily have inferior standing [36-40].

\section{Conclusion}

For PUMC, the expression of metabolism-related proteins such as Glut-1 and SDHB is likely different in tumor or stroma depending on the clinical and histologic subtype. SQ showed high expression for Glut-1 in tumor, AD for
SDHB in stroma and PD for CAIX in stroma. Higher Glut-1 expression was noted in the nodal type and lower Glut-1 expression in carcinomatosis type.

\section{Additional file}

Additional file 1: Table S1. Clone, dilution, and source of antibodies used.

\section{Abbreviations}

PUMC: Primary unknown metastatic carcinoma; AD: Adenocarcinomas; PD: Poorly differentiated carcinomas; SQ: Squamous cell carcinomas; UD: Undifferentiated carcinomas; FFPE: Formalin-fixed, paraffin-embedded; H\&E: Hematoxylin and eosin; TMA: Tissue microarray;

IHC: Immunohistochemistry; CA: Carbonic anhydrase; MCT: Monocarboxylate transporter; GLS1: Glutaminase1; GDH: Glutamate dehydrogenase; ASCT2: Amino acid transporter-2; SDH: Succinate dehydrogenase.

\section{Competing interests}

The authors declare that they have no competing interests.

\section{Authors' contributions}

HMK participated in the design of the study and performed the statistical analysis and drafted the manuscript. DHK carried out the immunochemistry. WHJ participated in its design. JSK conceived the study, and participated in its design and coordination and helped to draft the manuscript. All authors read and approved the final manuscript.

\section{Acknowledgements}

This research was supported by the Basic Science Research Program through the National Research Foundation of Korea (NRF) funded by the Ministry of Education, Science and Technology (2012R1A1A1002886).

Received: 23 September 2013 Accepted: 27 December 2013 Published: 6 January 2014 


\section{References}

1. Pavlidis N, Fizazi K: Cancer of unknown primary (CUP). Crit Rev Oncol Hematol 2005, 54:243-250.

2. Haskell CM, Cochran AJ, Barsky SH, Steckel RJ: Metastasis of unknown origin. Curr Probl Cancer 1988, 12:5-58.

3. Krementz ET, Cerise EJ, Foster DS, Morgan LR Jr: Metastases of undetermined source. Curr Probl Cancer 1979, 4:4-37.

4. Lembersky BC, Thomas LC: Metastases of unknown primary site. Med Clin North Am 1996, 80:153-171.

5. van de Wouw AJ, Jansen RL, Speel EJ, Hillen HF: The unknown biology of the unknown primary tumour: a literature review. Ann Oncol 2003, 14:191-196.

6. Warburg O: On the origin of cancer cells. Science 1956, 123:309-314.

7. Moreno-Sanchez R, Rodriguez-Enriquez S, Marin-Hernandez A, Saavedra E: Energy metabolism in tumor cells. FEBS J 2007, 274:1393-1418.

8. Wise DR, Thompson CB: Glutamine addiction: a new therapeutic target in cancer. Trends Biochem Sci 2010, 35:427-433.

9. Won KY, Kim GY, Kim YW, Song JY, Lim SJ: Clinicopathologic correlation of beclin-1 and bcl-2 expression in human breast cancer. Hum Pathol 2010, 41:107-112.

10. Airley R, Loncaster J, Davidson S, Bromley M, Roberts S, Patterson A, Hunter R, Stratford I, West C: Glucose transporter glut-1 expression correlates with tumor hypoxia and predicts metastasis-free survival in advanced carcinoma of the cervix. Clin Cancer Res 2001, 7:928-934.

11. Cantuaria G, Fagotti A, Ferrandina G, Magalhaes A, Nadji M, Angioli R, Penalver M, Mancuso S, Scambia G: GLUT-1 expression in ovarian carcinoma: association with survival and response to chemotherapy. Cancer 2001, 92:1144-1150.

12. Endo M, Tateishi U, Seki K, Yamaguchi U, Nakatani F, Kawai A, Chuman H, Beppu Y: Prognostic implications of glucose transporter protein-1 (glut-1) overexpression in bone and soft-tissue sarcomas. Jpn J Clin Oncol 2007, 37:955-960.

13. Kato H, Takita J, Miyazaki T, Nakajima M, Fukai Y, Masuda N, Fukuchi M, Manda R, Ojima H, Tsukada K, Kuwano H: Glut-1 glucose transporter expression in esophageal squamous cell carcinoma is associated with tumor aggressiveness. Anticancer Res 2002, 22:2635-2639.

14. Legan M, Luzar B, Marolt VF: Expression of cyclooxygenase-2, glucose transporter- 1 and angiogenesis in gallbladder carcinomas and their impact on prognosis. Scand J Gastroenterol 2009, 44:1101-1108.

15. Pizzi S, Porzionato A, Pasquali C, Guidolin D, Sperti C, Fogar P, Macchi V, De Caro R, Pedrazzoli S, Parenti A: Glucose transporter-1 expression and prognostic significance in pancreatic carcinogenesis. Histol Histopathol 2009, 24:175-185.

16. Stackhouse BL, Williams $H$, Berry $P$, Russell $G$, Thompson $P$, Winter JL, Kute T: Measurement of glut-1 expression using tissue microarrays to determine a race specific prognostic marker for breast cancer. Breast Cancer Res Treat 2005, 93:247-253.

17. Tateishi U, Yamaguchi U, Seki K, Terauchi T, Arai Y, Hasegawa T: Glut-1 expression and enhanced glucose metabolism are associated with tumour grade in bone and soft tissue sarcomas: a prospective evaluation by [18F]fluorodeoxyglucose positron emission tomography. Eur J Nucl Med Mol Imaging 2006, 33:683-691.

18. Eckert AW, Lautner MH, Taubert H, Schubert J, Bilkenroth U: Expression of glut-1 is a prognostic marker for oral squamous cell carcinoma patients. Oncol Rep 2008, 20:1381-1385.

19. Oliver RJ, Woodwards RT, Sloan P, Thakker NS, Stratford IJ, Airley RE: Prognostic value of facilitative glucose transporter glut- 1 in oral squamous cell carcinomas treated by surgical resection; results of EORTC translational research fund studies. Eur J Cancer 2004, 40:503-507.

20. Pavlides S, Whitaker-Menezes D, Castello-Cros R, Flomenberg N, Witkiewicz AK, Frank PG, Casimiro MC, Wang C, Fortina P, Addya S, et al: The reverse warburg effect: aerobic glycolysis in cancer associated fibroblasts and the tumor stroma. Cell Cycle 2009, 8:3984-4001.

21. Nguyen XC, So Y, Chung JH, Lee WW, Park SY, Kim SE: High correlations between primary tumours and loco-regional metastatic lymph nodes in non-small-cell lung cancer with respect to glucose transporter type 1-mediated 2-deoxy-2-F18-fluoro-D-glucose uptake. Eur J Cancer 2008, 44:692-698.

22. Kobayashi M, Kaida H, Kawahara A, Hattori S, Kurata S, Hayakawa M, Hirose Y, Uchida M, Kage M, Fujita H, et al: The relationship between GLUT-1 and vascular endothelial growth factor expression and 18F-FDG uptake in esophageal squamous cell cancer patients. Clin Nucl Med 2012, 37:447-452.

23. Abrams HL, Spiro R, Goldstein N: Metastases in carcinoma; analysis of 1000 autopsied cases. Cancer 1950, 3:74-85.

24. Gilbert $H$, Kagan A: Fundamental aspects of metastasis. Amsterdam: North-Holland Publishing; Weiss L; 1976.

25. Nystrom JS, Weiner JM, Heffelfinger-Juttner J, Irwin LE, Bateman JR, Wolf RM: Metastatic and histologic presentations in unknown primary cancer. Semin Oncol 1977, 4:53-58.

26. Le Chevalier T, Cvitkovic E, Caille P, Harvey J, Contesso G, Spielmann M, Rouesse J: Early metastatic cancer of unknown primary origin at presentation: a clinical study of 302 consecutive autopsied patients. Arch Intern Med 1988, 148:2035-2039.

27. Yamada T, Uchida M, Kwang-Lee K, Kitamura N, Yoshimura T, Sasabe E, Yamamoto T: Correlation of metabolism/hypoxia markers and fluorodeoxyglucose uptake in oral squamous cell carcinomas. Oral Surg Oral Med Oral Pathol Oral Radiol Endod 2012, 113:464-471.

28. Kim S, Kim do H, Jung WH, Koo JS: Expression of glutamine metabolism-related proteins according to molecular subtype of breast cancer. Endocr Relat Cancer 2013, 20:339-348.

29. Pavlides S, Tsirigos A, Vera I, Flomenberg N, Frank PG, Casimiro MC, Wang C, Fortina P, Addya S, Pestell RG, et al: Loss of stromal caveolin-1 leads to oxidative stress, mimics hypoxia and drives inflammation in the tumor microenvironment, conferring the "reverse Warburg effect": a transcriptional informatics analysis with validation. Cell Cycle 2010, 9:2201-2219.

30. Chang H, Shyu KG, Lee CC, Tsai SC, Wang BW, Hsien Lee Y, Lin S: GL331 inhibits HIF-1alpha expression in a lung cancer model. Biochem Biophys Res Commun 2003, 302:95-100.

31. Yeo EJ, Chun YS, Cho YS, Kim J, Lee JC, Kim MS, Park JW: YC-1: a potential anticancer drug targeting hypoxia-inducible factor 1. J Natl Cancer Inst 2003, 95:516-525.

32. Mohanti BK, Rath GK, Anantha N, Kannan V, Das BS, Chandramouli BA, Banerjee AK, Das S, Jena A, Ravichandran R, et al: Improving cancer radiotherapy with 2-deoxy-D-glucose: phase I/II clinical trials on human cerebral gliomas. Int J Radiat Oncol Biol Phys 1996, 35:103-111.

33. Aft RL, Zhang FW, Gius D: Evaluation of 2-deoxy-D-glucose as a chemotherapeutic agent: mechanism of cell death. Br J Cancer 2002, 87:805-812.

34. Vullo D, Franchi M, Gallori E, Pastorek J, Scozzafava A, Pastorekova S, Supuran CT: Carbonic anhydrase inhibitors: inhibition of the tumor-associated isozyme IX with aromatic and heterocyclic sulfonamides. Bioorg Med Chem Lett 2003, 13:1005-1009.

35. Gallagher SM, Castorino JJ, Wang D, Philp NJ: Monocarboxylate transporter 4 regulates maturation and trafficking of $C D 147$ to the plasma membrane in the metastatic breast cancer cell line MDA-MB-231. Cancer Res 2007, 67:4182-4189.

36. Dova L, Pentheroudakis G, Golfinopoulos V, Malamou-Mitsi V, Georgiou I, Vartholomatos G, Ntemou A, Fountzilas G, Pavlidis N: Targeting c-KIT, PDGFR in cancer of unknown primary: a screening study for molecular markers of benefit. J Cancer Res Clin Oncol 2008, 134:697-704.

37. Pavlidis N, Briassoulis E, Bai M, Fountzilas G, Agnantis N: Overexpression of C-myc, Ras and C-erbB-2 oncoproteins in carcinoma of unknown primary origin. Anticancer Res 1995, 15:2563-2567.

38. Hainsworth JD, Lennington WJ, Greco FA: Overexpression of Her-2 in patients with poorly differentiated carcinoma or poorly differentiated adenocarcinoma of unknown primary site. J Clin Oncol 2000, 18:632-635

39. van de Wouw AJ, Jansen RL, Griffioen AW, Hillen HF: Clinical and immunohistochemical analysis of patients with unknown primary tumour. A search for prognostic factors in UPT. Anticancer Res 2004, 24:297-301.

40. Briasoulis E, Tsokos M, Fountzilas G, Bafaloukos D, Kosmidis P, Samantas E, Skarlos D, Nicolaides C, Pavlidis N: Bcl2 and p53 protein expression in metastatic carcinoma of unknown primary origin: biological and clinical implications. A Hellenic Co-operative Oncology Group study. Anticancer Res 1998, 18:1907-1914.

doi:10.1186/1479-5876-12-2

Cite this article as: Kim et al:: Metabolic phenotypes in primary unknown metastatic carcinoma. Journal of Translational Medicine 2014 12:2. 